\title{
Individualizando la Violencia Escolar: Análisis de Prácticas Discursivas en una Escuela Municipal de la Región de Valparaíso
}

\author{
Individualizing School Violence: Analysis of Discursive \\ Practices in a Public School in the Region of Valparaíso
}

\author{
Verónica López, Claudia Carrasco, Macarena Morales, Álvaro Ayala, \\ Joedith López y Michelle Karmy \\ Pontificia Universidad Católica de Valparaíso
}

\begin{abstract}
Este estudio indagó las prácticas discursivas que sustentan la alta percepción de violencia escolar en escuelas municipalizadas de Chile. Se realizó un estudio de caso en una escuela básica municipal de nivel socioeconómico medio-bajo de la Región de Valparaíso. Participaron estudiantes de $7^{\circ}$ básico, sus profesores, apoderados, directivos, asistentes de la educación, auxiliares y otros informantes clave. Las técnicas de recolección de información fueron entrevistas en profundidad, observaciones no participantes y análisis de documentos. El análisis del discurso mostró formas de acción social orientadas a individualizar la violencia escolar, invisibilizar el rol de la escuela y externalizar las atribuciones de responsabilidad, desde el rol docente al equipo multidisciplinario, a través de la derivación interna de "niños-problema". Estas prácticas se retroalimentan para formar un ciclo de exclusión escolar que resta a los estudiantes oportunidades de participación en los espacios de aprendizaje al interior de la escuela.
\end{abstract}

Palabras clave: violencia escolar, bullying, discurso, exclusión escolar

This study investigated the discursive practices that sustain the high perception of school violence in Chilean public schools. A case study was performed in an elementary municipal school of mediumlow socioeconomic status of the Region of Valparaíso. Participants were $7^{\text {th }}$ grade students, their teachers, parents, school leaders, and support staff. Interviews, non participatory observations, and document analysis were used. Discourse analyses showed forms of social action orientated towards individualizing school violence, making the role of the school invisible, and externalizing the attributions of responsibility, from teachers to the multidisciplinary team, through internal referral of "difficult children". These practices are mutually complementary and form a cycle of school exclusion that reduces students' opportunities to participate in learning spaces within the school.

Keywords: school violence, bullying, discourse, school exclusion

La escuela debiese ser un espacio donde los niños construyan aprendizajes académicos y socioemocionales y aprendan a convivir de manera democrática, convirtiéndose en los protagonistas de sociedades más justas y participativas (Organización de las Naciones Unidas para la Educación, la Ciencia y la Cultura [UNESCO], 2002). Vivir en espacios libres de violencia es importante, no solo para la convivencia democrática, sino también para el bienestar psicológico y social (Páez \& Martin-Beristain, 2011).

Chile lleva más de una década recogiendo la percepción de altos niveles de violencia escolar. El estudio realizado por Arancibia (1994) detectó que en niveles socioeconómicos (NSE) bajos un $75 \%$ de la población percibía violencia entre los alumnos y un $43 \%$, abusos de parte de los profesores. Diez años después el estudio nacional sobre convivencia escolar solicitado por el

Verónica López, Claudia Carrasco, Macarena Morales, Álvaro Ayala, Joedith López y Michelle Karmy, Escuela de Psicología, Facultad de Filosofía y Educación, Pontificia Universidad Católica de Valparaíso, Viña del Mar, Chile.

Este estudio fue financiado por FONDECYT (Proyecto 11080055) y por PIA-CONICYT (Proyecto CIE-05).

La correspondencia relativa a este artículo debe ser dirigida a Verónica López, Escuela de Psicología, Facultad de Filosofía y Educación, Pontificia Universidad Católica de Valparaíso, Avda. El Bosque 1290, Viña del Mar, Chile. E-mail: veronica.lopez@ucv.cl 
Ministerio de Educación de Chile y UNESCO (Idea, 2005) reveló que el 87\% de los estudiantes y el $92 \%$ de los docentes refería agresión en la convivencia escolar de sus establecimientos.

La Tercera Encuesta Nacional de Violencia en el Ámbito Escolar (Chile, Ministerio del Interior, 2011) controló de manera más específica la agresión persistente entre compañeros (bullying) de otras formas de agresión. Los resultados indicaron una frecuencia de bullying —entendido como hostigamiento permanente y discriminación- de 14,5\%, cifra que está dentro de los rangos esperados de acuerdo a los estudios de prevalencia a nivel internacional. En un estudio regional, López, Morales y Ayala (2009) encontraron una frecuencia de 9\% de agresión persistente en el tiempo entre estudiantes (bullying), cifra similar al último estudio nacional.

Estos resultados sugieren que, aun cuando la opinión pública tiende a homologar los conceptos de bullying y violencia escolar, la alta percepción de violencia en las escuelas se refiere a algo más que a bullying. Según Benbenishty y Astor (2005), la violencia escolar está inserta (embedded) en los contextos locales y sociales de manera tal que las características de la cultura, comunidad local, familia y escuela ejercen mutuas influencias en la agresión entre escolares y pueden constituir fuentes de otro tipo de violencia en la escuela.

Esta manera de entender la violencia escolar se condice con lo que Noel (2008) ha denominado violencias en la escuela: la violencia no es única, sino multiforme y situada en un contexto determinado. Las formas de violencia involucran acciones de agresión directa, indirecta y simbólica, intra e inter-estamentos, de maneras más y menos explícitas. Así, no todas las formas de violencia son igualmente probables en todos los contextos sociales (Domènech \& Íñiguez, 2002), lo cual se vuelve crucial al momento de investigar en torno al tema, pues cada vez que nos encontramos frente a fenómenos de violencia, el componente subjetivo aparece como delimitador del espacio simbólico en que se enjuiciará una acción como violenta. Según Hernández (2005), es necesario comprender cómo la violencia es significada en diferentes contextos. Para la autora, es el espacio relacional donde emerge la violencia y se asienta culturalmente, por lo que "es en este espacio donde se puede comprender la dinámica de la violencia, al poder captar cómo sus propios efectos pasan a consolidarse cultural y estructuralmente, repotenciando los circuitos de violencia" (p. 62). Para ello, es necesario descubrir la violencia (Hernández, 2005). Si la violencia es creada en la interacción humana, entonces, se reproduciría en la intersubjetividad social.

A partir de lo anterior, destaca el papel de la escuela como un espacio social y simbólico que promueve situaciones mediatas o inmediatas que permiten el desencadenamiento de la acción violenta (Brown \& Munn, 2008; Martín-Baró, 1999). Para algunos investigadores (Flores \& Zerón, 2007; García \& Madriaza, 2005) el contexto escolar chileno actual estaría favoreciendo la emergencia de prácticas de violencia, por cuanto habría una pérdida del sentido de la educación tras el fracaso de las escuelas públicas en cumplir las promesas de integración social, afectando especialmente a los sectores más desfavorecidos de nuestra sociedad. Es en este sentido que Debarbieux (2003) propone a la violencia escolar como síntoma de las inequidades de la globalización, emergiendo, entonces, la relación entre escuela y sociedad en el análisis de la violencia en la escuela (Riella, 1999).

Charlot y Emin (1997) se refieren a los conceptos de violencia física, incivilidad y violencia simbólica y los sitúan en grados ascendentes de abstracción. Por violencia física se refieren a golpes, robos y otros actos de vandalismo, mientras que la incivilidad se caracteriza principalmente por lo que denominan microviolencias, compuestas por humillaciones y faltas de respeto. La violencia simbólica es una forma de violencia centrada en las relaciones de poder a través de estructuras sociales. Para Bourdieu y Passeron (1972) esta sería una violencia suave y eficaz, llevando a los dominados a plegar sus intereses, pensamientos y sentimientos a favor de los dominantes. Para estos autores el trabajo de adhesión al orden social establecido opera a través de diversos sistemas sociales, siendo uno de ellos el sistema escolar formal, basado en acciones pedagógicas múltiples, difusas e institucionales, capaces de sustituir la violencia física y psicológica en violencia simbólica.

Desde este enfoque, la función de la escuela sería la inculcación y apropiación de la cultura de la clase dominante, determinada en formas de conductas, saberes y disposiciones perdurables, contribuyendo a la reproducción de las desigualdades (Bourdieu \& Passeron, 1972). Para que la violencia simbólica sea efectiva requiere que quienes padecen violencia simbólica no sean capaces de detectarla, lo que implica un complejo entramado de relaciones sociales (Charlot \& 
Emin, 1997). Desde este marco de referencia, la violencia en el espacio escolar se puede entender, no solo como una violencia en las escuelas - a través de formas de agresión física, verbal y de exclusión social-, sino también de las escuelas, mediante prácticas simbólicas de reproducción de estructuras sociales.

Ante la pregunta ¿qué existe aparte del bullying o acoso escolar persistente entre estudiantes que permita explicar la mayor percepción de victimización de estudiantes de escuelas municipalizadas? y bajo la consideración del espacio intersubjetivo construido en y desde la escuela en la emergencia de la violencia escolar, se vuelve relevante la pregunta por las prácticas cotidianas discursivas. Desde una perspectiva discursiva pragmática (Wetherell \& Potter, 1996), el discurso es acción y, siéndolo, es capaz de constituir procesos de violencia simbólica, toda vez que los hablantes producen ciertos sentidos que construyen visiones de mundo y utilizan recursos lingüísticos tendientes a legitimar los órdenes de mundo de los grupos de poder y privilegio, bajo la complicidad del discurso dominante (Gorski, 2011, Abril). Fue este el foco de análisis del estudio que reportamos.

\section{Método}

\section{Diseño}

Llevamos a cabo una investigación de carácter cualitativo, con el objetivo de identificar y caracterizar las prácticas cotidianas simbólicas y discursivas en la interacción entre los actores de la escuela que inciden en la violencia escolar en el NSE medio-bajo. Optamos por un estudio de caso, proceso de indagación que se caracteriza por el examen detallado, comprehensivo, sistemático y en profundidad del caso objeto de interés (García Jiménez, 1988). Siguiendo las recomendaciones de Rodríguez, Gil y García (1998), el caso que presentamos corresponde al entorno educativo de los estudiantes de una escuela básica municipal de la Región de Valparaíso, Chile, considerada como una única unidad de análisis.

\section{Participantes}

Siguiendo a Rodríguez et al. (1998), los criterios que deben perseguir la selección del caso no se plantean en términos de representatividad de los mismos, ya que la investigación cualitativa centra su preocupación en los elementos subjetivos e idiosincráticos. Stake (1994) propone ciertos criterios de selección del caso que fueron incorporados en este estudio: tener fácil acceso al caso; alta probabilidad de que exista una mezcla de procesos, programas, personas, interacciones y/o estructuras relacionadas con las preguntas de investigación; establecer una buena relación con los informantes; la posibilidad de que el investigador pueda desarrollar su rol durante todo el tiempo que sea necesario; y asegurar la calidad y credibilidad del estudio.

De este modo, realizamos el estudio en el establecimiento educacional antes mencionado, el cual, según datos del Sistema de Medición de Calidad de la Educación de Chile, es de NSE medio-bajo. Tiene orientación laica y atiende niveles de enseñanza pre-básica y básica, distribuidos en dos jornadas (mañana y tarde, sin jornada escolar completa). Al momento del estudio, la matrícula era de 851 estudiantes distribuidos en 28 cursos, $1^{\circ}$ a $8^{\circ}$ básico en tres paralelos (con un promedio de 31 alumnos por curso) y dos paralelos de kinder y pre-kinder. La escuela tiene Proyecto de Integración para estudiantes con necesidades educativas especiales e implementa el Plan de Acción de la Ley de Subvención Escolar Preferencial (SEP). Seleccionamos este establecimiento tras su participación en un estudio previo sobre caracterización de maltratos entre pares estudiantes mediante cuestionarios de autoinforme y heteroinforme (López et al., 2009), realizado por el mismo equipo de investigación. En dicho estudio, la escuela había obtenido un nivel medio de percepción de intimidación por parte de los estudiantes. De manera independiente a nuestro estudio, el equipo de gestión de la escuela había aplicado la medición del Sistema de Alerta Temprana de Violencia Escolar el año anterior (2009), cuyo resultado arrojó un nivel medio-bajo de agresión, en comparación a otros establecimientos municipalizados de la misma comuna. 
Respecto de la infraestructura del establecimiento, en un terreno de $1300 \mathrm{~m}^{2}$ se encuentran un edificio de cemento de dos pisos y un patio central no techado. En el primer nivel está la mayoría de las salas, que son utilizadas por dos cursos, uno en la jornada de la mañana y otro en la jornada de la tarde. En este nivel se encuentran el casino y los espacios administrativos, como la oficina de Inspectoría General, de Dirección, de la Orientadora, la sala de Profesores y la del Equipo Multidisciplinario. Las integrantes de este último (una psicóloga, dos educadoras diferenciales y una orientadora) fueron contratadas con recursos de la Ley SEP. Existen tres salas recientemente acondicionadas para atención de apoderados y una nueva sala construida para un curso de primer ciclo, lo que implicó quitar espacio al patio central. Dicho patio es el espacio común recreativo de los estudiantes y también es utilizado para las ceremonias y actividades deportivas. En el segundo nivel están emplazados el Centro de Recursos (biblioteca-sala de consejo), la sala Enlaces (computadores con conexión a Internet) y una sala de clases. Desde el establecimiento se han realizado negociaciones con el sostenedor municipal para ampliar su infraestructura inmobiliaria, con el objetivo de atender adecuadamente a la cantidad de estudiantes matriculados.

Focalizamos el estudio en los tres cursos de $7^{\circ}$ año básico, llevando a cabo un muestreo de tipo teórico (Glaser \& Strauss, 1967). Seleccionamos este nivel por cuanto nuestro estudio cuantitativo precedente arrojó como resultado que dichos cursos tenían una frecuencia de agresiones entre estudiantes mayor que el resto (López et al., 2009). Buscamos incluir como participantes, además, a informantes clave que interactuaban directamente con estos estudiantes: sus apoderados, sus profesores, los directivos, asistentes de educación, auxiliares y profesionales de apoyo psicosocial. Los criterios para la selección de cada individuo siguieron un muestreo por voluntarios y en cadena (Salamanca \& Martín-Crespo, 2007). En la Tabla 1 describimos a los participantes según tipo de actor educativo.

\section{Técnicas de Recolección de Datos}

Siguiendo los principios de la investigación cualitativa, consideramos los datos como producciones discursivas (Sisto, 2008), asumiendo que el investigador forma parte de la realidad investigada y co-participa en su producción. Utilizamos un conjunto de técnicas narrativas, así como observaciones y técnicas documentales.

Entrevistas en profundidad. Elaboramos un guión de entrevista, siguiendo la modalidad de entrevista focalizada (Alonso, 1999), el que aplicamos de manera individual y grupal. Las preguntas indagaron en los significados y apreciaciones de los participantes acerca de la violencia y la convivencia escolar, complementándolas con hechos concretos registrados por los investigadores en las observaciones no participantes.

Observaciones no participantes. A pesar de que las observaciones fueron no participantes, las realizamos involucrando la interacción social entre los investigadores y los informantes, recogiendo datos de modo sistemático y no intrusivo. En este sentido, los investigadores debimos someternos a las reglas formales e informales del grupo observado, participando en diversos momentos y accediendo a la cotidianidad del grupo, desarrollando lo que Pérez Serrano (1994) denomina observación participante pasiva. Con ellas realizamos registros narrativos y diarios del investigador. Mientras que los primeros sistematizan eventos conductuales, los segundos corresponden a informes personales que recogen las interpretaciones de los investigadores sobre la continuidad y discontinuidad de dichos eventos. Algunas observaciones de aula las grabamos en audio y las transcribimos y complementamos con las notas del registro narrativo, construyendo lo que Gómez y Prieto (1996) denominan registro ampliado de aula. Los elementos de interés durante las observaciones eran las formas de interacción intra e inter estamentos, así como los patrones discursivos que fueron posibles de identificar en estas interacciones. El foco estuvo puesto en los contenidos explícitos así como en las estrategias discursivas (legitimaciones, deslegitimaciones, señalizaciones a estudiantes, entre otras). 
Recolección de documentos. Para la selección de los documentos seguimos las recomendaciones de Prieto (2001), recopilando lo que la autora denomina documentos oficiales, en este caso, Proyecto Educativo Institucional (PEI), Manual de Convivencia, libros de clases, protocolos de atención a estudiantes y comunicaciones escritas por profesores en las Libretas de Comunicación de los estudiantes.

Realizamos las observaciones y entrevistas durante dos semanas consecutivas, acompañando a la escuela durante la jornada escolar desde el inicio hasta el final de la misma, participando, de esta manera, de los espacios cotidianos y naturales producidos en la escuela. En este período de tiempo también recolectamos los documentos oficiales. En la Tabla 1 mostramos las técnicas que empleamos con cada tipo de participante.

Tabla 1

Participantes y Técnicas de Recolección de Datos

\begin{tabular}{|c|c|c|c|c|}
\hline Tipo de participantes & $\begin{array}{c}\mathrm{N}^{\circ} \text { de } \\
\text { participantes }\end{array}$ & $\begin{array}{c}\mathrm{N}^{\mathrm{o}} \text { de } \\
\text { entrevistas }\end{array}$ & $\begin{array}{l}\mathrm{N}^{o} \text { y tipo } \\
\text { documentos }\end{array}$ & $\begin{array}{c}\mathrm{N}^{\mathrm{o}} \text { de } \\
\text { observaciones }\end{array}$ \\
\hline $\begin{array}{l}\text { Equipo de gestión educativa } \\
\text { (directora, inspector, jefe de } \\
\text { Unidad Técnica Pedagógica } \\
\text { [UTP]) }\end{array}$ & 3 & $\begin{array}{l}1 \text { grupal a los } 3 \\
\text { integrantes }\end{array}$ & $\begin{array}{l}\text { PEI } \\
\text { Reglamento interno }\end{array}$ & $\begin{array}{l}1 \text { directora } \\
2 \text { inspector } \\
1 \text { jefe de UTP }\end{array}$ \\
\hline $\begin{array}{l}\text { Equipo multidisciplinario } \\
\text { (orientadora, } 2 \text { educadoras } \\
\text { diferenciales, psicóloga) }\end{array}$ & 4 & $\begin{array}{l}2 \text { educ. diferenciales } \\
1 \text { psicóloga }\end{array}$ & Ficha de derivación & $\begin{array}{l}1 \text { equipo completo ( } 4 \\
\text { participantes) } \\
2 \text { orientadora }\end{array}$ \\
\hline Profesores jefes de $7^{\circ}$ básico & 3 & 1 profesor $7^{\circ} \mathrm{C}$ & $\begin{array}{l}\text { Libro de clases } 7^{\circ} \mathrm{A} \\
\text { Libro de clases } 7^{\circ} \mathrm{B} \\
\text { Libro de clases } 7^{\circ} \mathrm{C}\end{array}$ & $\begin{array}{l}1 \text { reg. ampliado } 7^{\circ} \mathrm{A} \\
1 \text { reg. ampliado } 7^{\circ} \mathrm{B} \\
1 \text { reg. ampliado } 7^{\circ} \mathrm{C}\end{array}$ \\
\hline $\begin{array}{l}\text { Profesores de asignatura } 7^{\circ} \\
\text { básicos }\end{array}$ & 4 & 1 profesor $7^{\circ} \mathrm{A}$ & $\begin{array}{l}\text { Libro de clases } 7^{\circ} \mathrm{A} \\
\text { Libro de clases } 7^{\circ} \mathrm{B} \\
\text { Libro de clases } 7^{\circ} \mathrm{C}\end{array}$ & $\begin{array}{l}2 \text { profesores } 7^{\circ} \mathrm{A} \\
1 \text { profesora } 7^{\circ} \mathrm{B} \\
1 \text { profesora } 7^{\circ} \mathrm{C}\end{array}$ \\
\hline $\begin{array}{l}\text { Estudiantes de } 7^{\circ} \text { básico ( } 3 \\
\text { paralelos) }\end{array}$ & $\begin{array}{l}7^{\circ} \mathrm{A}: 35 \\
7^{\circ} \mathrm{B}: 35 \\
7^{\circ} \mathrm{C}: 32\end{array}$ & $\begin{array}{l}1 \text { grupal (5 } \\
\text { estudiantes) } \\
2 \text { alumnas } 7^{\circ} \mathrm{A} \\
1 \text { alumna } 7^{\circ} \mathrm{B} \\
1 \text { alumno } 7^{\circ} \mathrm{C} \\
1 \text { alumno } 7^{\circ} \mathrm{D}\end{array}$ & $\begin{array}{l}3 \text { libretas de } \\
\text { comunicaciones }\end{array}$ & $\begin{array}{l}1 \text { reg. ampliado } 7^{\circ} \mathrm{A} \\
1 \text { reg. ampliado } 7^{\circ} \mathrm{B} \\
1 \text { reg. ampliado } 7^{\circ} \mathrm{C}\end{array}$ \\
\hline $\begin{array}{l}\text { Asistentes de la Educación con } \\
\text { funciones de Paradocentes }\end{array}$ & 5 & 2 asistentes & & $\begin{array}{l}1 \text { observación a } 5 \\
\text { asistentes en el } \\
\text { recreo }\end{array}$ \\
\hline $\begin{array}{l}\text { Madres, padres y apoderados de } \\
7^{\circ} \text { básico }\end{array}$ & $\begin{array}{l}7^{\circ} \mathrm{A}: 35 \\
7^{\circ} \mathrm{B}: 35 \\
7^{\circ} \mathrm{C}: 32\end{array}$ & $\begin{array}{l}1 \text { apoderado } 7^{\circ} \mathrm{A} \\
1 \text { apoderado } 7^{\circ} \mathrm{B}\end{array}$ & & $\begin{array}{l}1 \text { reunión } 7^{\circ} \mathrm{A} \\
1 \text { reunión } 7^{\circ} \mathrm{B} \\
1 \text { reunión } 7^{\circ} \mathrm{C} \\
3 \text { citaciones a } \\
\text { apoderados }\end{array}$ \\
\hline $\begin{array}{l}\text { Asistentes de la Educación con } \\
\text { funciones de Auxiliares }\end{array}$ & 4 & $\begin{array}{l}1 \text { grupal a los } 4 \\
\text { integrantes }\end{array}$ & & 1 observación \\
\hline $\begin{array}{l}\text { Programa Habilidades Para la } \\
\text { Vida (2 psicólogas) }\end{array}$ & 2 & $\begin{array}{l}1 \text { grupal a las } 2 \\
\text { integrantes }\end{array}$ & & $\begin{array}{l}1 \text { observación de } \\
\text { taller a docentes }\end{array}$ \\
\hline $\begin{array}{l}\text { Monitores centro comunal de } \\
\text { violencia escolar }\end{array}$ & 4 & $\begin{array}{l}1 \text { grupal a los } 4 \\
\text { integrantes }\end{array}$ & & \\
\hline Centro de Estudiantes & 1 & 1 individual & & \\
\hline $\begin{array}{l}\text { Centro de Madres, Padres y } \\
\text { Apoderados }\end{array}$ & 3 & $\begin{array}{l}1 \text { grupal a } 3 \\
\text { integrantes }\end{array}$ & & \\
\hline
\end{tabular}




\section{Procedimiento}

Una vez obtenida la autorización del establecimiento, solicitamos el consentimiento informado de todos los actores participantes. En el caso de los estudiantes, solicitamos además el consentimiento informado de sus padres. Dos de los tres profesores jefes de $7^{\circ}$ básico no quisieron participar en las entrevistas individuales, pero sí accedieron a participar de las observaciones de aula. En consideración de los aspectos éticos, este artículo resguarda el anonimato de la escuela y sus participantes. Las entrevistas y observaciones fueron realizadas en los horarios destinados por el establecimiento a sus actividades. Los documentos oficiales fueron proporcionados por el equipo de gestión, el equipo multidisciplinario y por los estudiantes.

\section{Análisis de la Información}

Analizamos la información por medio del análisis del discurso pragmático (Edwards, Potter \& Middleton, 1992). Este tipo de análisis supone que el discurso es acción, en tanto produce efectos que "vehiculan ciertos significados, sentidos, órdenes de mundo" (Íñiguez, 2003, p. 109). La finalidad del análisis fue indagar en las prácticas lingüísticas presentes en la escuela en torno al abordaje de la violencia escolar que mantienen y promueven ciertas relaciones sociales. Para el análisis seguimos las propuestas de Wetherell y Potter (1996) en relación a la identificación y análisis de repertorios interpretativos (RI), entendidos como "los elementos esenciales que los hablantes utilizan para construir versiones de las acciones, los procesos cognitivos y otros fenómenos" (p. 113). Los RI corresponden a una gama restringida de técnicas usadas bajo un estilo y gramática específicos, con el fin de construir hipótesis sobre los propósitos y consecuencias del lenguaje (Wetherell \& Potter, 1996). Ya que la regularidad del discurso no se puede probar a nivel del hablante individual, se analizan todos los discursos, escritos y hablados, con el fin de identificar las consistencias e inconsistencias del discurso, las que, para los autores, son diferencias entre unidades lingüísticas relativamente vinculadas e internamente consistentes, dando origen a los RI (Wetherell \& Potter, 1996).

\section{Resultados}

Desarrollamos el análisis en torno a tres RI: la individualización de la violencia escolar, la invisibilización del rol de la escuela y la externalización de la responsabilidad. Estos repertorios corresponden a formas restringidas de acción social que demarcan ciertos sentidos y producen una gama limitada de relaciones sociales. Estas formas de acción social se vinculan entre sí, manteniendo y produciendo la exclusión escolar. De este modo, el eje temático que articula las categorías de análisis es el ciclo de exclusión escolar, que se representa en la Figura 1. Un cuarto y último repertorio pone en tensión este ciclo de exclusión escolar y permite prever elementos para la transformación: la visibilización del rol de la escuela. A continuación describimos y detallamos estos RI. 


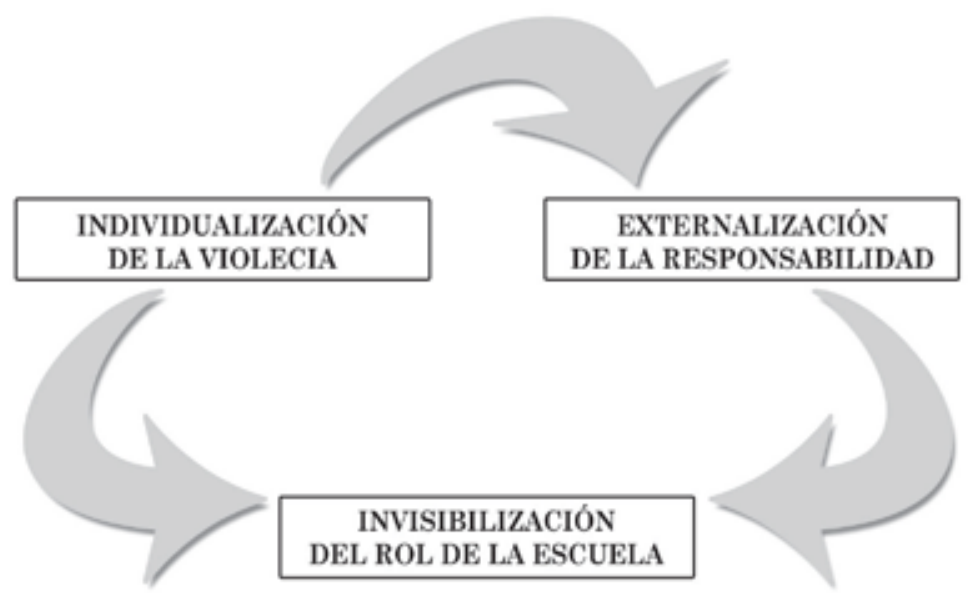

Figura 1. El ciclo de exclusión escolar en el abordaje de la violencia escolar.

\section{Individualizar la Violencia Escolar: el "Caso"}

En el discurso de distintos actores de la escuela se observa una construcción del sujeto estudiante que tiende hacia una caracterización negativa de este, particularmente desde los profesores. Ellos son vistos como individuos con carencias, las que se materializarían en conductas que se alejan de la norma, destacando un discurso ideal implícito frente al cual son comparados. Para estos agentes educativos dicha visión obedece en gran parte a la dependencia municipal de la escuela. La estrategia discursiva que opera es la legitimación de la visión estigmatizada del estudiante de escuela municipal:

A ver, yo pienso que hay buena convivencia acá. A pesar que es un colegio municipalizado, se supone que los colegios municipalizados están encasillados en que son niños problemas, niños con dificultad. (Entrevista profesora)

Esta visión carenciada del estudiante, centrada en el déficit, permite que los docentes se posicionen en un rol de "salvador", llamado a completar las carencias en el trabajo con estudiantes de dependencia municipal. Esta mirada tendría matices paternalistas, los que son reconocidos y validados por estos actores educativos:

Si yo te digo, nosotros somos psicólogos, orientadores, profesores, mamás, de todo... (Entrevista profesora)

Acto del día lunes, 10 de la mañana, después del primer recreo. Los profesores están parados arriba y los alumnos abajo, en el patio. Habla una profesora, los está invitando a portarse bien: "ya que ustedes en sus casas no les dan valores, nosotros se los vamos a dar. Por eso necesito que ustedes entiendan..." (Extracto cuaderno de campo)

Están acostumbrados a que se les den las cosas hechas, que tú les indiques lo que tienen que hacer y prácticamente se lo hagas. Entonces que él tenga que hacer algo, que él tenga que pensar, que él tenga que trabajar, a ellos les da flojera. (Entrevista profesora)

Por su parte, el equipo de gestión construye añoranzas de una época pasada, en la que la educación era considerada un privilegio. La consecuencia de este discurso es alejar a la educación de su condición de derecho, junto con responsabilizar principalmente al propio estudiante y a su esfuerzo por los resultados de aprendizaje, invisibilizando así el papel de las condicionantes sociales: 
(...) un perfil de alumno que era diferente al perfil que tenemos ahora, más comprometido. (Entrevista Equipo de Gestión)

Por lo tanto, la gente que quería un buen nivel de sus alumnos los traía y se comprometía a todo el nivel de exigencia. (Entrevista Equipo de Gestión)

En la interacción profesor-alumnos en la sala de clases prevalece el castigo de conductas por sobre el diálogo o la conversación. En el aula se castigan aquellas conductas que evidencian que el estudiante ha dejado de centrar su atención en el docente. Esto es reconocido por los apoderados y principalmente por los propios estudiantes. La consecuencia de esta práctica implica lo que Foucault (2002) denomina la sanción normalizadora: una vigilancia que permite calificar, clasificar y castigar:

No vamos a gastar más tiempo en eso, en hacer silencio, y vamos simplemente a mandar "al tiro"

a inspectoría. A ver, silencio, por favor. (Registro ampliado sala de clases $7^{\circ}$ básico)

Para otorgar mayor consistencia a este RI, los profesores tienden a atribuir intencionalidad a las conductas cuestionadas, suponiendo que por medio de estos comportamientos se desafía a la autoridad pedagógica. Esto se puede evidenciar en las anotaciones del Registro del Desarrollo Escolar del libro de clases de un $7^{\circ}$ básico. En él se consigna, además de las conductas, una supuesta intencionalidad a la base. Como acto lingüístico, esto se vuelve una declaración, que vehiculiza la generación de un mundo en el cual los estudiantes pierden el tiempo, son despreocupados y no se comprometen (ver Figura 2).

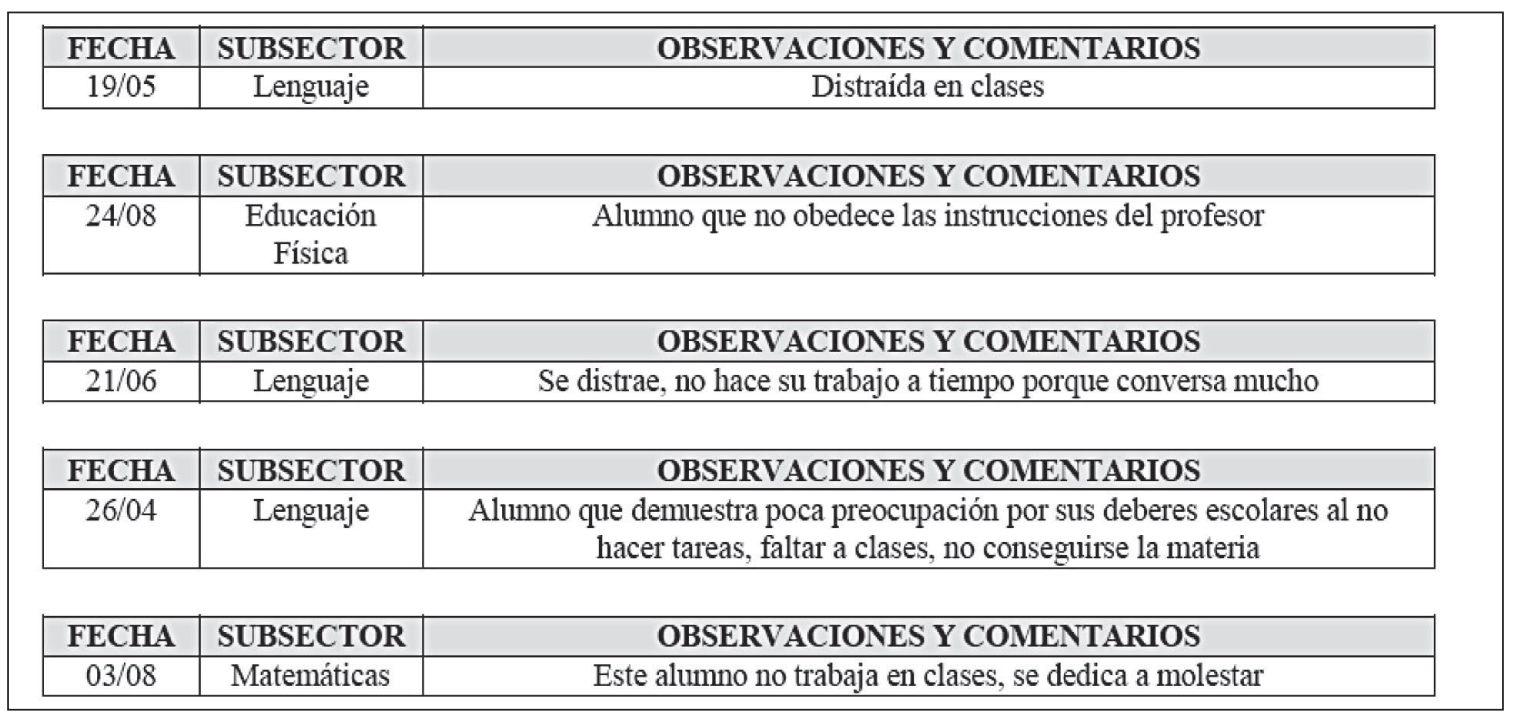

Figura 2. Extractos de un libro de clases de $7^{\circ}$ básico, correspondiente a la sección Registro de Desarrollo Escolar.

Profesores, equipo multidisciplinario y equipo de gestión identifican a algunos estudiantes que tienen más problemas de conducta que otros, los cuales denominan "casos". Hablar de "casos" o "alumnos problema" es una estrategia discursiva que permite a los hablantes circunscribir el problema de la violencia escolar a ciertos niños específicos. El efecto que esto tiene es restringir las acciones de abordaje a la intervención específica con esos niños. Resulta importante visualizar cómo esta conceptualización influye en la mirada y el abordaje del equipo psicosocial en el trabajo con los estudiantes:

(...) yo entré a la sala de clases y, antes de entrar, la profesora me dijo: "Mira, este es desordenado, este es desordenado, este es desordenado" y yo, probablemente dentro de una actividad, voy 
a tratar de que ese desordenado se integre mejor a la actividad de todos. (Entrevista psicólogas Programa Habilidades para la Vida)

Este RI muestra cómo la validación de las declaraciones existe solo si se someten a la debida autoridad, lo que garantiza su eficacia. Así, nos encontramos con una calificación de las conductas y una contabilidad de individuos basadas en valores opuestos del bien y el mal. Esta contabilidad convierte a los estudiantes con menor apego a la norma como un "caso", vale decir, como un individuo cuya conducta hay que encauzar o corregir (Foucault, 2002). Se trataría del lugar que se ocupa en una clasificación que, a su vez, individualiza los cuerpos por una localización que los distribuye y los hace circular en un sistema de relaciones. Por otra parte, el RI de individualización a través del "caso" remite al "caso clínico" y, mediante ello, a la autoridad del modelo médico como garante de una etiología patologizante.

\section{Invisibilizar el Rol de la Escuela}

Para profesores, equipo multidisciplinario y equipo directivo las causas de las carencias percibidas son atribuidas a factores externos e incontrolables por la escuela. En el discurso prevalece la negativización del medio cultural socioeconómico y el espacio físico del colegio, construyendo como declaración un determinismo ambiental en el proceso de adquisición de conductas. El espacio físico reducido es utilizado como argumento para explicar comportamientos agresivos, escasez de articulación en el trabajo docente y poca innovación pedagógica (ver Figuras 3 y 4).

El grupo de docentes es emprendedor, dispuesto al cambio, con bastantes horas de perfeccionamiento, pero de baja aplicabilidad en el aula. Persisten las clases frontales, metodología tradicional, poco innovadoras, atribuibles a la falta de espacio, lo que conlleva a un hacinamiento, por la gran cantidad de alumnos (961 alumnos en un terreno de 1300 metros cuadrados), los que asisten a doble jornada.

Figura 3. Extracto del PEI de la escuela (página 2).

Debilidades:

Espacio Físico.

Salas estrechas.

Mobiliario inadecuado.

Material didáctico insuficiente.

Apoderados poco comprometidos (50\%).

Hacinamiento (Exceso de alumnos).

\section{Amenazas:}

Entorno sociocultural descendido.

Problemas de drogradicción y alcoholismo.

Madres jefas de hogar, quienes se ausentan de casa todo el tiempo.

Hogares mal constituidos.

Figura 4. Extracto del PEI de la escuela (páginas 5 y 6). 
En el discurso docente se construye a los apoderados como uno de los principales responsables de la violencia, caracterizándolos como individuos con poca preocupación e interés por sus hijos. Esto está explicitado en el PEI (ver Figura 4) y forma parte de las prácticas discursivas producidas en las entrevistas y en las observaciones. Algunos apoderados contribuyen a confirmar estos juicios elaborados en la escuela:

Se ve poco, poco interés del apoderado y poco apoyo del apoderado. (Entrevista profesora)

Los apoderados no quieren participar en nada (...) los apoderados no están muy involucrados con los niños. (Entrevista presidenta Centro de Padres)

En las citas anteriores aparece la retórica de la renuncia (disclaim; Wetherell \& Potter, 1996), en cuanto los apoderados que construyen estas afirmaciones no se incluyen a sí mismos en el grupo descrito, aludiendo a los apoderados como si se tratara de un grupo al cual no pertenecen. De esta manera, se construye un ideal de familia que posibilita que los docentes recurran a autoconfirmar su identidad orientada a suplir la carencia, colocándose a sí mismos como los encargados de encauzar las conductas de los estudiantes, suponiendo que el papel normalizador de la familia habría fracasado. Este discurso es compartido por el equipo de gestión:

No, los valores hay que estarlos recalcando cada vez y el problema de acá, yo creo que es general.

Los valores tenemos que entregarlos nosotros, no lo entregan los papás. (Entrevista profesora)

(...) si ellos necesitan hablar conmigo, yo estoy, por cualquier cosa, yo ahi estoy, hagan cuenta que

soy la mamá, y yo muchas veces los aconsejo no siendo la mamá. (Entrevista profesora)

Un ejemplo de ello es la Hoja de Derivación que maneja el establecimiento (ver Figura 5). Este protocolo, creado por el equipo multidisciplinario, intenciona la búsqueda de aspectos negativos en las familias. La estrategia discursiva es, nuevamente, legitimar el discurso de carencia del estudiante y su familia.

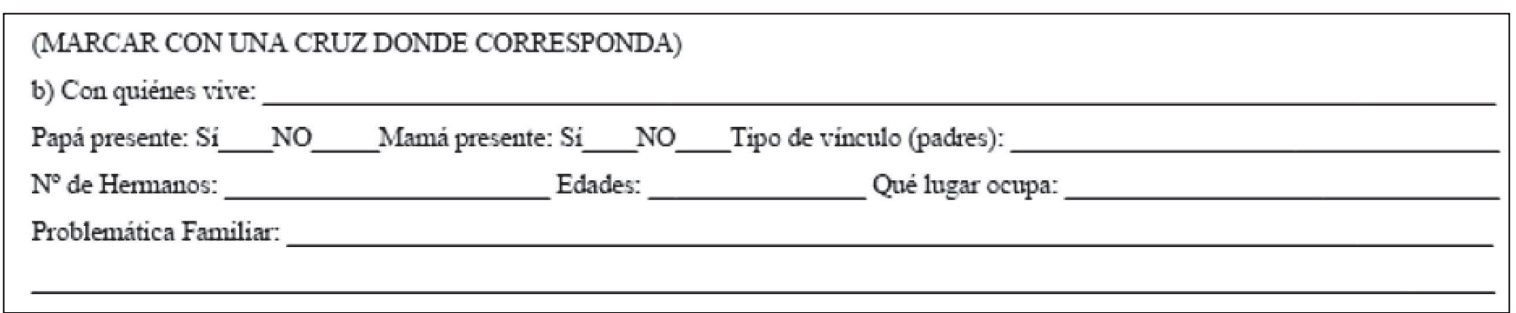

Figura 5. Extracto de la Hoja de Derivación.

De esta manera, se construye una visión de estudiantes que son confirmados como individuos problemáticos, lo que se justifica por medio de naturalizaciones que se vinculan con la dependencia municipal del establecimiento, con el exceso de estudiantes al que esta escuela atiende y, también, con la influencia de pares que son a su vez "negativizados". El recurso lingüístico utilizado es la argumentación, esgrimiendo los profesores y el equipo directivo las razones por las cuales la escuela poco puede hacer para abordar el problema, puesto que este no está en la escuela, sino en algunos estudiantes agresivos, que lo son debido a razones ajenas a la misma. El efecto de esta práctica, además de individualizar la violencia escolar, es tornar invisible el rol de la escuela en la emergencia del fenómeno.

\section{Externalizar la Responsabilidad Pedagógica}

Transformada la violencia escolar en un problema de "alumnos-caso" y sumada a una desvalorización del apoyo de las familias, el abordaje de la violencia escolar produce una forma de acción social focalizada en estos alumnos-problema, centrada en la sanción normalizadora hacia ellos y en la externalización de la responsabilidad de hacer frente a este tema.

En la escuela no observamos acciones sistemáticas de prevención de la violencia escolar generadas desde los profesores y/o el equipo de gestión. Las acciones de prevención que se 
llevan a cabo son gestionadas por la dupla de psicólogas del programa Habilidades Para la Vida, ambas sin permanencia en la escuela. En las instancias en que los equipos de gestión y multidisciplinario se hacen cargo de los "casos de problemas conductuales" lo hacen desde un enfoque centrado en el modelo médico. Establecen prácticas y procedimientos que operan para individualizar las causas del problema, situando este en el estudiante y su familia.

Opera una derivación dentro de la propia escuela, la que actúa como un primer mecanismo de exclusión escolar. Nótese en la siguiente cita cómo la profesora emplea el término médico derivar para referirse a la acción que define la relación entre ella y el equipo multidisciplinario:

Claro, y los derivan, o sea, también estamos nosotros ahí, pero también se deriva a la orientadora, se deriva al psicólogo, todo eso. (Entrevista profesora)

En esta cita es posible identificar, además, la variabilidad del discurso; "o sea, también estamos nosotros allí" se interpone como un contra-discurso que apela a la función pedagógica del profesor de aula para abordar la violencia escolar.

Entre los dispositivos que emplea el equipo multidisciplinario para individualizar el origen de los fenómenos considerados negativos en los estudiantes, y externalizar la responsabilidad de su atención, destacan la Hoja de Derivación y el Instrumento de Derivación. Ambos se concentran en las carencias de los estudiantes y sus familias, dejando de lado sus potencialidades y fortalezas. Esto refuerza la mirada del niño como un caso clínico, apareciendo listas de cotejo de características que se señalan como síntomas (ver Figuras 6 y 7 ).

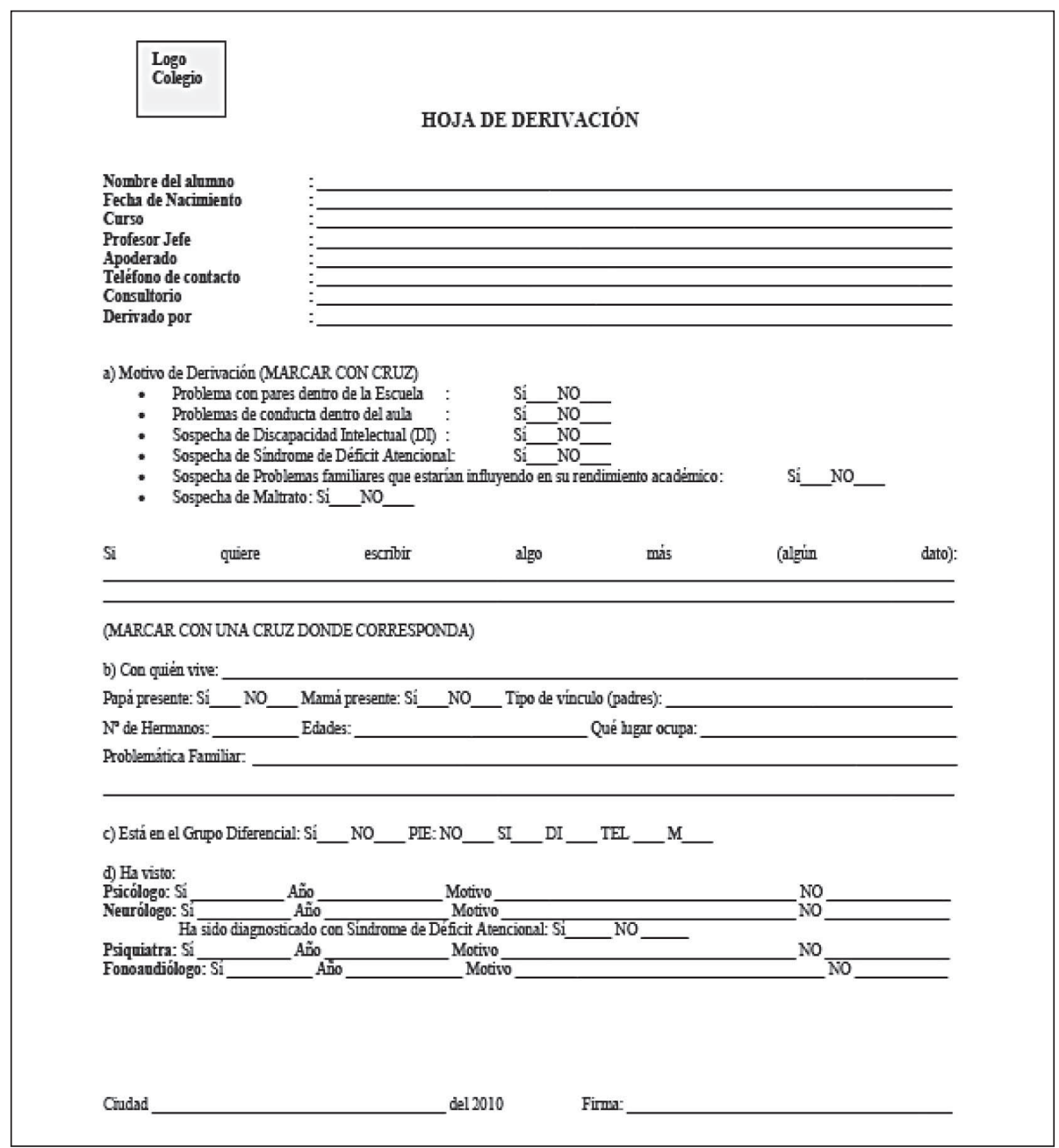

Figura 6. Hoja de Derivación (página 1). 
III.- PRINCIPALES SINTOMAS ASOCIADOS QUE SE DEBEN CONSIDERAR (marcar con una $X$ )

1

2.

3.

4.

5.

6.

7.

$8 . \longrightarrow$ dificultades en concentración

9. agresión verbal

10. —_ agresión fisica

11.

12.

13. baja tolerancia a la frustración

14. inseguridad en el aula

15. _ angustia en el actuar

¿DESDE CUÁNDO SE PRESENTAN LOS SINTOMAS?

\section{IV.- COMENTARIOS - SUGERENCIAS DESDE LA DERIVACIÓN}

Figura 7. Extracto de la Hoja de Derivación (página 2). La primera página consiste en la identificación del estudiante.

Al estar basadas en el modelo médico, las acciones que implementa el equipo multidisciplinario colaboran con la invisibilización de la escuela, en cuanto obvian cómo y en qué medida la relación profesor-estudiante y estudiante-sala de clases influyen en la emergencia de los problemas. Una de las consecuencias de esta forma de acción social es el etiquetamiento y la validación del ciclo de exclusión:

...es que, ese es el problema, es la costumbre. Es la costumbre, entonces, eso lleva a que muchas cosas también se hagan normales, po, se haga normal hablar de un niño, por ejemplo, que a mí me carga... en los desayunos, [hablar] acerca de los niños. No, que el niño tanto es así, es asá. Se hace normal develar situaciones como si [fuese] nada, ese niño "no, es que le pegan todos los días", "ese niño fue abusado". Con una simpleza, que tú dices... jestoy cansada! (Entrevista psicóloga)

En la cita anterior se puede apreciar una naturalización del discurso normalizador, en cuanto se le asigna a la costumbre la responsabilidad de las prácticas estigmatizadoras de estudiantes. Esta forma de hablar favorece la externalización de responsabilidad en relación con las causas del fenómeno, lo que se ve fortalecido con el hecho de ocultar gramaticalmente el sujeto en su discurso. Al referir que "se hace normal", la hablante utiliza la renuncia como retórica, lo que le permite no asumirse como participante activa de esta dinámica: 
En general eso es lo que esperan como los profes, cómo no hacerse mucho cargo, sino como que los saquen de la sala y que el niño vuelva distinto (...) es como la varita mágica. (Entrevista dupla psicosocial)

Lo anterior se evidencia con mayor claridad en los informes elaborados por la orientadora del establecimiento, quien, luego de emitirlos, espera que sea la psicóloga de la escuela quien se encargue del encauzamiento de las conductas de los estudiantes. De este modo, se considera a la psicología como la última opción, dotándola del poder de transformar conductas. Con esto se construye el supuesto de que las conductas aparecen al margen de los procesos educativos.

En el siguiente extracto del informe pedagógico de un estudiante, elaborado por la orientadora del establecimiento, se evidencia la dinámica de exclusiones y externalizaciones: si no se acata la sanción normalizadora, se externaliza hacia la psicóloga del establecimiento, quien queda, entonces, a cargo de esta normalización (ver Figura 8).

\footnotetext{
Con los profesores es frontal y contestatario, se altera y descontrola fácilmente, responsabiliza a sus pares de sus actos, por lo que tiene poca tolerancia a las llamadas de atención que estos le hacen. No cambia sus actitudes una vez que los docentes se lo han solicitado, incluso cuando estos le han dado más de una oportunidad para modificarse. Conducta reactiva.
}

Con el niño se hicieron compromisos de conducta y responsabilidad. Mediación con los compañeros y compañeras con los que tenía más conflicto.

Las sanciones que implicaban participar en algunas actividades se le hacian día a día de acuerdo a su comportamiento actual.

Se conversó con él, con los profesores y alumnos, para comprometerlos a ayudarle.

\section{IV.- CONCLUSIONES}

Las estrategias acordadas no dieron resultados. Su familia no adopta las sugerencias dadas, por lo que el alumno sigue viniendo sin útiles y tareas. Su conducta no varió en nada.

Se derivó a Psicóloga.

Figura 8. Extracto del informe pedagógico de la orientadora sobre un estudiante.

\section{Visibilizar el Rol de la Escuela}

Los RI anteriores operan a través de prácticas discursivas que se han vuelto cotidianas para los equipos profesionales de la escuela y que se vuelven declarativas, construyendo un mundo social coherente. A pesar de dicha cotidianeidad, aparece en los enunciados de estudiantes y apoderados un RI de inconsistencia, como es de esperarse en un análisis de discurso pragmático. Algunos apoderados y estudiantes afirman que estos procedimientos llegan al límite del maltrato, tanto en la arbitrariedad como en la severidad con que son ejercidos:

(...) De repente el niño como que, ya, 'ta bien que [el profesor] lo rete. Pero de repente no decirle, no sé po, de repente, como decir, no sé qué ejemplo buscarte, pero de repente se pasan en molestar a los alumnos. Dicen cosas como muy fuertes. (Entrevista estudiante)

Mi hijo P (...) aquí en el colegio no me ayudan con él, siempre dicen que está planeando algo, alguna maldad, entonces, como que me lo etiquetan. (Cuaderno de Campo, reunión apoderados)

Estos discursos permiten un nivel simbólico de contradicción, en los que se ubica la posibilidad de transformación de las prácticas de exclusión social. En cuanto afirmaciones, solo son posibles de emitir como discurso cuando existen componentes culturales comunes que les dan sentido (Wetherell \& Potter, 1996). A través de estas prácticas lingüísticas, se desnaturaliza la violencia y se visibiliza el rol de la escuela, ofreciendo alternativas a la externalización de responsabilidad. 


\section{Discusión}

Los resultados revelan que en la escuela operan prácticas de estigmatización del estudiante municipal, individualización de la violencia escolar y externalización de la responsabilidad del rol docente, a través de procedimientos institucionalizados de derivación interna de niñosproblema.

$\mathrm{Al}$ naturalizar las causas de los fenómenos educativos no deseados, se atribuye a características individuales la responsabilidad de las conductas no deseadas por la escuela, "psicologizando" y reduciendo así el análisis del fenómeno educativo (Baltar, 2003). La caracterización negativa de los estudiantes en la escuela estudiada opera fundamentalmente como un instrumento de control social, por cuanto se encarga de imponer un discurso ideal construido por los hablantes como inalcanzable por los estudiantes. Estas ideologías de déficit (deficit ideologies) se entienden como prácticas sistemáticas que tienden a concebir a los estudiantes desde sus carencias y déficits y, en última instancia, a "patologizar" sus dificultades (Ahlquist, 2011, Abril), cumpliendo la función de justificar sistemas existentes de poder y privilegio, junto con racionalizar las inequidades existentes (Gorski, 2011, Abril).

El énfasis en el castigo de conductas que se desvían de la norma - la obediencia a la autoridad pedagógica - da cuenta del valor social del poder disciplinario (Foucault, 2002), cuyo éxito, en este caso, radicaría en el uso de instrumentos, como la sanción normalizadora y la construcción del estudiante como un caso. De este modo, aparece el componente disciplinario como parte de la violencia institucional ejercida por la escuela (Riella, 1999). Siguiendo a Bourdieu y Passeron (1972), se estaría cumpliendo con la función de inculcación y apropiación del arbitrario cultural. Sin embargo, en esta escuela se evidencian las consecuencias sociales cada vez que esta función no consigue los resultados esperados, permitiendo que ingrese un cuestionamiento simbólico a la capacidad de integración del actual orden social (Riella, 1999). Si los docentes y asistentes de la educación fallan en su cometido de adaptar a los estudiantes, comienza a activarse un mecanismo de exclusión que termina por despojar a la escuela de su papel formador, fortaleciendo prácticas de discriminación (Magendzo, 1996, Junio) que se evidencian en la siguiente dinámica: El profesor de aula intenta corregir conductas no deseadas; al no lograrlo, deriva al estudiante a la orientadora como si este fuese un caso. Luego, la orientadora, junto a las educadoras diferenciales, elaboran un informe pedagógico que, al estar basado en el modelo médico, prescribe un tratamiento individual focalizado en el niño o niña y su familia. Si con esto no logra los resultados esperados, se deriva al estudiante a la psicóloga, último eslabón del equipo multidisciplinario, quien, finalmente, es la que debe hacerse cargo de la modificación conductual (Bahía Bock, 2003).

Proponemos que las prácticas de invisibilización, individualización y externalización se retroalimentan y potencian entre sí, llegando a formar un ciclo de exclusión escolar. Sostenemos que se trata de un ciclo de exclusión escolar, puesto que la cadena de derivación interna y todos los procedimientos institucionalizados para ello no permiten a estos estudiantes participar en los espacios de aprendizaje al interior del aula. Si el propósito de la educación es formar ciudadanos preparados para las exigencias del siglo XXI (UNESCO, 2008), la educación de calidad involucra no solo aprendizajes, sino también participación, la que se les resta a estos estudiantes. Con esto se le hace sentir al estudiante que es merecedor de esta exclusión, debido a componentes psicológicos de su personalidad, despojándolo de su capacidad de desarrollo de autonomía. Para Parsons (2005) las políticas de castigo y exclusión de la escuela están arraigadas culturalmente y se constituyen como prácticas tendientes a ubicar la responsabilidad en los individuos, sus familias o el fracaso social.

En este estudio buscamos identificar y comprender los discursos presentes en la escuela, desde distintos actores educativos, siendo la diversidad de participantes una de sus riquezas. Constatamos que el discurso de individualización y externalización se construye fundamentalmente desde los profesores y el equipo de gestión, usando como recursos la legitimación y argumentación; pero participan también los profesionales de apoyo psicosocial y los apoderados, a través de la retórica de la renuncia y la naturalización. Es en la voz de los estudiantes donde 
es posible identificar fisuras en el discurso dominante, que apela al papel de la escuela en la emergencia y en las alternativas de solución de la violencia.

El propósito de este artículo no ha sido culpar a la escuela, sino comprender las dinámicas discursivas que vehiculizan ciertos sentidos y otorgan ejes para ordenar y hacer coherentes ciertas visiones de mundo (Íñiguez, 2003). Concluimos que los RI dificultan que la escuela se involucre en acciones de prevención y promoción, al no narrarse como parte del problema. Cabe preguntarse ¿qué posibilidades tiene la escuela de transformarse a sí misma y modificar este ciclo de exclusión escolar? La cuarta categoría de análisis arroja luces al respecto: hacer visible el papel de la escuela involucra procesos de concientización (Freire, 1993). En este estudio fueron algunos de los estudiantes y apoderados quienes tuvieron una visión de la violencia escolar vinculada con responsabilidades docentes, aunque tímidamente dejan entrever su propia responsabilidad.

Para superar el ciclo de exclusión al abordar la violencia escolar, concluimos que será necesario revisar y reflexionar sobre las visiones que sustentan esta exclusión, así como sobre las retóricas discursivas que las construyen y mantienen. Para cuestionar el ciclo de exclusión que se genera en la violencia escolar, al parecer, es necesario cuestionar la función social de la escuela.

Entendemos la individualización como la consecuencia del poder de normalización que obliga a la homogeneidad, pero individualiza, al permitir las desviaciones con el fin de retroalimentar la función de normalización (Foucault, 2002). Así, la constatación de las prácticas discursivas y simbólicas de individualización, invisibilización y externalización que hemos analizado, ofrecen la posibilidad de llevar a cabo un ejercicio de vigilancia epistemológica (Bourdieu, Chamboredon \& Passeron, 2002), tanto para profesores y directivos como para los profesionales de apoyo psicosocial.

Una limitación del estudio es que el diseño de estudio de caso y la tradición paradigmática que lo sustenta no permite realizar generalizaciones a la población; no obstante, la matriz cultural compartida hace pensar que estas retóricas no son exclusividad de esta escuela. Sostenemos que es posible a futuro cuantificar la cadena de externalización y exclusión que se produce en las escuelas, a partir de las prácticas de derivación. Para ello será necesario estar atentos a las prácticas de orientadores y psicólogos: ¿Qué porcentaje de su tiempo dedican a acciones orientadas a la atención individual de alumnos versus el trabajo con profesores? ¿Qué espacios ocupan mayoritariamente en la escuela, la sala de atención individual o la sala de clases? Estos indicadores serán relevantes para ampliar las mediciones de calidad de la educación, de manera que incluyan, además del rendimiento escolar, elementos como la participación, la inclusión y el clima escolar. Pero, además, será necesario desarrollar otras estrategias metodológicas que superen la individualización y externalización, propiciando el trabajo con y desde los profesores de aula.

\section{Referencias}

Ahlquist, R. (2011, Abril). Teacher education for the public good: Subverting hyper-accountability and deficit discourse in social justice teacher education. Ponencia presentada en la 2011 Annual Meeting of the American Educational Research Association, New Orleans, Estados Unidos.

Alonso, L. E. (1999). Sujeto y discurso: el lugar de la entrevista abierta en las prácticas de la sociología cualitativa. En L. E. Alonso (Ed.), La mirada cualitativa en sociología: una aproximación interpretativa (pp. 67-92). Madrid, España: Fundamentos.

Arancibia, V. (1994). La educación en Chile: percepciones de la opinión pública y de expertos. Estudios Públicos, 54, 125-150.

Bahía Bock, A. (2003). Psicología de la educación: complicidad ideológica. En A. Bahía Bock, A. Checcia \& M. Rebello De Souza (Eds.), Psicología escolar: teorías críticas (pp. 79-103). São Paulo, Brasil: Casa do Psicólogo.

Baltar, M. J. (2003). El sentido del diagnóstico psicológico escolar. Un análisis crítico y una propuesta en construcción. Psicoperspectivas, 2(1), 7-34.

Benbenishty, R. \& Astor, R. A. (2005). School violence in context: Culture, neighborhood, family, school, and gender. New York, NY: Oxford University Press.

Bourdieu, P., Chamboredon J. C. \& Passeron, J. C. (2002). El oficio de sociólogo: presupuestos epistemológicos. Ciudad de México, México: Siglo XXI. 
Bourdieu, P. \& Passeron, J. (1972). La reproducción: elementos para una teoría del sistema de enseñanza. Barcelona, España: Laia.

Brown, J. \& Munn, P. (2008). 'School violence' as a social problem: Charting the rise of the problem and the emerging specialist field. International Studies in Sociology of Education, 18, 219-230. doi:10.1080/09620210802492807

Charlot, B. \& Emin, J. C. (1997). La violence à l'école: État des saviors [La violencia en la escuela: estado del arte]. Paris, Francia: Armand Colin.

Chile, Ministerio del Interior (2011). III Encuesta Nacional de Violencia en el Ámbito Escolar. Santiago, Chile: Autor, División de Seguridad Pública.

Debarbieux, E. (2003). School violence and globalization. Journal of Educational Administration, 41, 582-602. doi:10. 1108/09578230310504607

Domènech, M. \& Íñiguez, L. (2002). La construcción social de la violencia. Athenea Digital, 2, Material 2.

Edwards, D., Potter, J. \& Middleton, D. (1992). Toward a discursive psychology of remembering. The Psychologist, 5, 441-446.

Flores, L. \& Zerón, A. M. (2007). Paradojas de la violencia escolar en Chile: desde la subjetividad fenomenológica de los actores. Boletín de Investigación Educacional, 22, 14-28.

Foucault, M. (2002). Vigilar y castigar. Madrid, España: Siglo XXI.

Freire, P. (1993). Pedagogía de la esperanza, un encuentro con la pedagogía del oprimido. Ciudad de México, México: Siglo XXI.

García, M. \& Madriaza, P. (2005). Sentido y sinsentido de la violencia escolar: análisis cualitativo del discurso de estudiantes chilenos. Psykhe, 14(1), 165-180. doi:10.4067/S0718-22282005000100013

García Jiménez, E. (1988). Una teoría práctica sobre la evaluación. Revista de Educación (Ministerio de Educación de España), 287, 233-253.

Glaser, B. G. \& Strauss, A. L. (1967). The discovery of grounded theory: Strategies for qualitative research. Chicago, IL: Aldine de Gruyter.

Gómez, F. \& Prieto, M. (1996). Metodologías activo-participativas. Valparaíso, Chile: Pontificia Universidad Católica de Valparaíso.

Gorski, P. C. (2011, Abril). Poverty, class, and the nature of deficit ideology in U. S. schooling. Ponencia presentada en la 2011 Annual Meeting of the American Educational Research Association, New Orleans, Estados Unidos.

Hernández, T. (2005). Des-cubriendo la violencia. En R. Briceño-León, (Comp.), Violencia, sociedad y justicia en América Latina (pp. 57-75). Buenos Aires, Argentina: Facultad Latinoamericana de Ciencias Sociales.

Idea (2005). Informe Final Primer Estudio Nacional de Convivencia Escolar. Santiago, Chile: Ministerio de Educación/ Organización de las Naciones Unidas para la Educación, la Ciencia y la Cultura.

Íñiguez, L. (2003). Análisis del discurso: manual para las ciencias sociales. Barcelona, España: Universitat Oberta de Catalunya.

López, V., Morales, M. \& Ayala, A. (2009). Maltrato entre pares: conductas de intimidación y victimización en escolares chilenos. Revista de Psicología de la Pontificia Universidad Católica del Perú, 27, 243-286.

Magendzo, A. (1996, Junio). Discriminaciones en la educación: un tema insoslayable para la educación democrática. Ponencia presentada en el Seminario Prejuicios, Estereotipos y Discriminaciones en la Educación, Santiago, Chile.

Martín-Baró, I. (1999). Acción e ideología. Ciudad de México, México: McGraw Hill.

Noel, G. (2008). Versiones de la violencia: las representaciones nativas de la violencia y su reconstrucción analítica en las escuelas de barrios populares urbanos. Propuesta Educativa, 30, 101-108.

Organización de las Naciones Unidas para la Educación, la Ciencia y la Cultura (2002). Cultura de paz en la escuela: mejores prácticas en la prevención y tratamiento de la violencia escolar. Santiago, Chile: Autor, Oficina Regional de Educación de la UNESCO para América Latina y el Caribe.

Organización de las Naciones Unidas para la Educación, la Ciencia y la Cultura (2008). Los aprendizajes de los estudiantes de América Latina y El Caribe. Primer reporte del Segundo Estudio Regional Comparativo y Educativo (SERCE). Santiago, Chile: Autor, Oficina Regional de Educación de la UNESCO para América Latina y el Caribe.

Páez, D. \& Martín-Beristain, C. (2011). Superando la violencia colectiva y construyendo cultura de paz. Madrid, España: Fundamentos.

Parsons, C. (2005). School exclusion: The will to punish. British Journal of Educational Studies, 53, $187-211$. doi:10.1111/j.1467-8527.2005.00290.x

Pérez Serrano, G. (1994). Investigación cualitativa: retos e interrogantes. Madrid, España: La Muralla.

Prieto, M. (2001). La investigación en el aula: ¿una tarea posible? Valparaíso, Chile: Ediciones Universidad Católica de Valparaíso.

Riella, A. (1999). Violencia y control social: el debilitamiento del orden social de la modernidad. Revista Ciencias Sociales, Universidad La República, Uruguay, 16, 5-20.

Rodríguez, G., Gil, J. \& García, E. (1998). Metodología de la investigación cualitativa. Granada, España: Aljibe.

Salamanca, A. \& Martín-Crespo, C. (2007). El muestreo en la investigación cualitativa. Nure Investigación, 27, 1-4. Extraído de http://www.nureinvestigacion.es/FICHEROS_ADMINISTRADOR/F_METODOLOGICA/ FMetodologica_27.pdf

Sisto, V. (2008). La investigación como una aventura de producción dialógica: la relación con el otro y los criterios de validación en la metodología cualitativa contemporánea. Psicoperspectivas, 7(1), 114-136. 
Stake, R. (1994). Investigación con estudio de casos. Madrid, España: Morata.

Wetherell, M. \& Potter, J. (1996). El análisis del discurso y la identificación de los repertorios interpretativos. En A. J. Gordo \& J. L. Linaza (Comps.), Psicologías, discursos y poder (pp. 63-78). Madrid, España: Visor.

Fecha de recepción: Abril de 2011.

Fecha de aceptación: Octubre de 2011. 